\title{
ON CONVEX FUNCTIONS OF ORDER $\alpha$ AND TYPE $\beta$
}

\author{
WANCANO MA \\ Department of Mathematics \\ Northwest University \\ Xian, China
}

(Received Apri1 14, 1987 and in revised form June 1, 1987)

ABSTRACT. Owa [1] gave three subordination theorems for convex functions of order $\alpha$ and starlike functions of order a. Unfortunately, none of the theorems 1s correct. In this paper, similar problems are discussed for a generalized class and sharp results are given.

KEY WORDS AND PHRASES. Subordination, Hadamard product, convex functions of order $\alpha$ and type $B$.

1980 AMS SUBJECT CLASSIFICATION CODE. $30 \mathrm{C} 45$.

\section{INTRODUCTION.}

Let $f(z)$ and $g(z)$ be analytic in the unit disk $D=\{z:|z|<1\}$. $f(z)$ is said to be subordinate to $g(z)$, dentoed by $f(z) \prec g(z)$, If there exists a functinn w(z) analytic and satisfying $|w(z)| \leq|z|$ in $D$ such that $f(z)=g(w(z))$. SEPTEMBER 1988 valent in $D$, then $f(z) \prec g(z)$ is equivalent to $f(0)=g(0)$ and $f(D) \subset g(D)$.

Let $S *(\alpha, \beta)$ be the family of starlike functions of order $\alpha$ and type $\beta$. That is, it consists of analytic functions $f(z)=z+a_{2} z^{2}+\ldots$ satisfying

$$
\left|z f^{\prime}(z) / f(z)-1\right|<\left|(2 \beta-1) z f^{\prime}(z) / f(z)+1-2 \beta \alpha\right| \quad(z \in D),
$$

where $0 \leqq \alpha<1$ and $0<\beta \leqq 1$. This class was first introduced by Juneja and Mogra [2]. It is clear that $S^{*}(\alpha, 1)=S^{*}(\alpha)$, the usual class of starlike functions of order $\alpha$.

Similarly, we define the following general class.

DEFINITION. An analytic function $f(z)=z+a_{2} z^{2}+\ldots$ is called convex of order $\alpha$ and type $B$ if and only if

$$
\left|z f^{\prime \prime}(z) / f^{\prime}(z)\right|<\left|(2 \beta-1) z f^{\prime \prime}(z) / f^{\prime}(z)+2 \beta(1-\alpha)\right| \quad(z \in D) \text {. }
$$

The class of these functions is denoted by $\mathrm{k}(\alpha, \beta)$.

$K(\alpha, 1)$ is the well known class $K(\alpha)$, which consists of convex functions of order

$\alpha$. It is easily seen that $f(z) \in K(\alpha, \beta)$ if and only if $z f^{\prime}(z) \in S^{*}(\alpha, \beta)$.

We got the following theorem in [3].

THEOREM A. Let $f(z) \in S^{*}(\alpha, \beta)$, then we have

$$
z f^{\prime}(z) / f(z) \prec(1+(1-2 \beta \alpha) z) /(1+(1-2 \beta) z),
$$




$$
\begin{array}{ll}
f(z) / z \prec(1+(1-2 \beta) z)^{2 \beta(1-\alpha) /(1-2 \beta)} & \left(\beta \neq \frac{1}{2}\right), \\
f(z) / z \prec e^{(1-\alpha) z} & \left(\beta=\frac{1}{2}\right) .
\end{array}
$$

All of the results are best possible.

In section 2 of this paper, we give a counterexample of Owa's results and point out the mistakes in [1]. Then we discuss simflar problems for the class $K(\alpha, \beta)$ and get sharp subordination and convolution theorems. And we give a characterization for convex functions of order $\alpha$ and type $\beta$ in section 3. Finally, we obtain some inequalities by using the subordination results.

2. A COUNTEREXAMPLE.

Theorem 1 in [1] is equivalent to that if $f(z) \in K(\alpha)$, then

$$
f^{\prime}(z) \prec e^{-4(1-\alpha) /(1-z)}=F(z),
$$

and if $f^{\prime}\left(r e^{i \theta}\right)$ lies for some $r \neq 0$ on the boundary of $F(|z|<r)$ if and only if

$$
f(z)=\int_{0}^{z} e^{4(1-\alpha) /(1-\varepsilon t)} d t \quad(|\varepsilon|=1) .
$$

It is we11 known that

$$
f(z)=\int_{0}^{z}(1-t)^{-2(1-\alpha)} d t \in K(\alpha) .
$$

(2.1) Implies that

$$
(1-z)^{-2(1-\alpha)} \prec e^{-4(1-\alpha) /(1-z)},
$$

or equivalently,

$$
\log (1-z) \prec 2 /(1-z)
$$

where $\log$ is to be the branch which vanishes at the point one. But clearly, (2.3) does not hold.

The mistake arises from that

$$
z f^{\prime \prime}(z) / f^{\prime}(z) \prec 4(1-\alpha) z /(1-z)^{2}
$$

implies

$$
\log ^{\prime}(z) \prec-4(1-\alpha) /(1-z)
$$

In fact, from (2.4) we can only get

$$
\log ^{\prime}(z) \prec 4(1-\alpha) z /(1-z) .
$$

And (2.2) was got from $f(0)=f^{\prime}(0)-1=0$ and

$$
\mathrm{zf}^{\prime \prime}(\mathrm{z}) / \mathrm{f}^{\prime}(\mathrm{z})=4(1-\alpha) \varepsilon z /(1-\varepsilon z)^{2} .
$$

So it should be

$$
f(z)=\int_{0}^{z} \exp [4(1-\alpha) \varepsilon t /(1-\varepsilon t)] d t .
$$


Furthermore, this function does not belong to $K(\alpha)$.

There are similar mistakes in the theorem 2 [1]. And the family of functions which satisfy the conditions in theorem $3[1]$ is empty since $\operatorname{Re}\left\{z f^{\prime}(z)\right\}=0$ at $z=0$. Therefore this theorem is meaningless. From the proof of the theorem 3 , maybe the condition should be $\operatorname{Re}\left\{z f^{\prime}(z)\right\}<\alpha, \operatorname{not} \operatorname{Re}\left\{z f^{\prime}(z)\right\}>\alpha$. If so, the following proof goes wrong again. There are also several places needed to be corrected. We omit them here.

3. SUBORDINATION AND CONVOLUTION THEOREMS.

The leading element of $K(\alpha, \beta)$ is

$$
k(\alpha, \beta, z)= \begin{cases}(1-2 \beta \alpha)^{-1}\left\{(1+(1-2 \beta) z)^{(1-2 \beta \alpha) /(1-2 \beta)}-1\right\} & \left(\beta \neq \frac{1}{2}, \alpha \neq \frac{1}{2} / \beta\right) \\ (1-2 \beta)^{-1} \log (1+(1-2 \beta) z) & \left(\beta \neq \frac{1}{2}, \alpha=\frac{1}{2} / \beta\right)(3.1) \\ \left(e^{(1-\alpha) z}-1\right) /(1-\alpha) & \left(\beta=\frac{1}{2}\right)\end{cases}
$$

It is not difficult to prove that for an analytic function $f(z)=z+a_{2} z^{2}+\ldots$, (1.3) implies that $f(z) \in S^{*}(\alpha, \beta)$. From Theorem $A$ and the correspondence between $K(\alpha, \beta)$ and $S^{*}(\alpha, \beta)$, we have the following

THEOREM 1. $f(z) \in K(\alpha, \beta)$ if and only if $f(z)=z+a_{2} z^{2}+\ldots$ is analytic in $D$ and

$$
z f^{\prime \prime}(z) / f^{\prime}(z) \downarrow 2 \beta(1-\alpha) z /(1+(1-2 \beta) z)
$$

Moreover, let $f(z) \in K(\alpha, \beta)$, then we have sharp subordinations

$$
\begin{array}{ll}
f^{\prime}(z) \prec(1+(1-2 \beta) z)^{2 \beta(1-\alpha) /(1-2 \beta)} & \left(\beta \neq \frac{1}{2}\right), \\
f^{\prime}(z) \prec e^{(1-\alpha) z} & \left(\beta=\frac{1}{2}\right) .
\end{array}
$$

The first result of Theorem 1 is equivalent to that an analytic function $f(z)=z+a_{2} z^{2}+\ldots \in K(\alpha, \beta)$ if and only if

$$
1+z f^{\prime \prime}(z) / f^{\prime}(z) \in Q(\alpha, \beta) \quad(z \in D),
$$

where

$$
Q(\alpha, \beta)= \begin{cases}\left\{w ;|w-\alpha-(1-\alpha) / 2(1-\beta)|<\frac{1}{2}(1-\alpha) /(1-\beta)\right\} & (\beta<1) \\ \{w ; \text { Rew }>\alpha\} & (\beta=1) .\end{cases}
$$

COROLLARY 1. $K\left(\alpha, \beta_{1}\right) \subset K\left(\alpha, \beta_{2}\right) \subset K(\alpha, 1)=K(\alpha)$ if $\beta_{1} \leqq \beta_{2} \leqq 1$. $\mathrm{K}\left(\alpha_{1}, \beta\right) \subset \mathrm{K}\left(\alpha_{2}, \beta\right) \subset \mathrm{K}(0, \beta)$ if $\alpha_{1} \geqq \alpha_{2} \geqq 0$.

THEOREM 2. Let $\mathrm{p}(\mathrm{z}) \in \mathrm{K}=\mathrm{K}(0,1)$, and $\mathrm{f}(\mathrm{z}) \in \mathrm{K}(\boldsymbol{a}, \boldsymbol{\beta})$, then

$$
\mathrm{p} * \mathrm{f}(\mathrm{z}) \in \mathrm{K}(\alpha, \beta),
$$

where * denotes the Hadamard product.

PROOF. We know that $1+z f^{\prime \prime}(z) / f^{\prime}(z)=\left(z f^{\prime}(z)\right)^{\prime} / f^{\prime}(z)$ takes all its values in the convex domain $Q(\alpha, \beta)$. A result of Ruscheweyh and Sheil-Small [4] implies that 
$p(z) *\left\{z\left(z f^{\prime}(z)\right)^{\prime}\right\} / p(z) *\left(z f^{\prime}(z)\right)$ also takes all its values in $Q(\alpha, \beta)$ since we have $\mathrm{f}(\mathrm{z}) \in \mathrm{K}(\alpha, \beta) \subset \mathrm{K}(0,1)=\mathrm{K}$ from Corollary 1 . It is easy to see that

$$
\begin{aligned}
& p(z) *\left(z f^{\prime}(z)\right)=z(p * f)^{\prime}(z), \\
& p(z) *\left\{z\left(z f^{\prime}(z)\right)^{\prime}\right\}=z\left\{p(z) *\left(z f^{\prime}(z)\right)\right\}^{\prime}=z\left\{z(p * f)^{\prime}(z)\right\}^{\prime} \\
& = \\
& z(p * f)^{\prime}(z)+z^{2}(p * f)^{\prime \prime}(z) .
\end{aligned}
$$

Thus for each $z \in D$

$$
1+z(p * f)^{\prime \prime}(z) /(p * f)^{\prime}(z)=p(z) *\left\{z\left(z f^{\prime}(z)\right)^{\prime}\right\} / P(z) *\left(z f^{\prime}(z)\right) \in Q(\alpha, \beta),
$$

which yields $p * f(z) \in K(\alpha, \beta)$. The proof is completed.

For $\alpha=0$ and $\beta=1$, Theorem 2 is the well known Polya-Schoenberg conjecture proved in [4].

COROLLARY 2. $K(\alpha, \beta) \subset \mathrm{s}^{*}(\alpha, \beta)$.

PROOF. If $f(z) \in K(\alpha, \beta)$. Let $p(z)=\log (1-z)^{-1}$ in Theorem 2 , we get

$$
g(z)=\int_{0}^{z} f(t) / t d t \in K(\alpha, \beta),
$$

which gives that $f(z)=z^{\prime}(z) \in s^{*}(\alpha, \beta)$.

LEMMA 1. $G(z)=k(\alpha, \beta, z) / z k^{\prime}(\alpha, \beta, z)$ is an analytic and convex univalent function in D. Moreover, $G(z)$ is analytic and univalent on $\bar{D}$ except for $z=1$ when $\beta=1$ for which $\lim _{z \rightarrow 1} G(z)=\infty$.

$z \in D$

PROOF. We may assume that $\beta \neq \frac{1}{2}$ and $\alpha \neq \frac{1}{2} / \beta$ since the convexity for $\beta=\frac{1}{2}$ or $\alpha=\frac{1}{2} / \beta$ can be deduced from the convexity for $\beta \neq \frac{1}{2}$ and $\alpha \neq \frac{1}{2} / \beta$.

From (3.1), we find that

$$
G(z)=\left(2 \beta-1+G_{1}(z)\right) /(2 \beta \alpha-1),
$$

where

$$
G_{1}(z)=z^{-1}\left\{(1+(1-2 \beta) z)^{2 \beta(1-\alpha) /(2 \beta-1)}-1\right\}
$$

So we have

$$
G_{1}(z)+2 \beta(1-\alpha)=2 \beta(1-\alpha) / z \int_{0}^{z} G_{2}(t) d t,
$$

where

$$
G_{2}(z)=1-(1+(1-2 \beta) z)^{(1-2 \beta \alpha) /(2 \beta-1)}
$$

Hence

$$
1+z G_{2}^{\prime \prime}(z) / G_{2}^{\prime}(z)=(1-(1-2 \beta \alpha) z) /(1+(1-2 \beta) z),
$$

which yields that $G_{2}(z)$ is a convex univalent function. $G_{1}(z)+2 \beta(1-\alpha)$ is also convex follows from a result due to Libera [5]. Thus $G_{1}(z)$ is convex, and so is $G(z)$. This results in the conclusions as desired. When $\beta=1$, we can get the other result easily. We come to the end of our proof. 
THEOREM 3. Let $f(z) \in K(\alpha, \beta)$, we have sharp subordination

$$
z f^{\prime}(z) / f(z) \prec z k^{\prime}(\alpha, \beta, z) / k(\alpha, \beta, z)
$$

To prove Theorem 3, we need the following lemma due to Miller and Mocanu [6]. LEMMA A. Let $q(z)=a+q_{1} z+\ldots$ be regular and univalent on $\vec{D}$ except for those points $\zeta \in \partial D$ for which $\lim _{z \rightarrow \zeta, z \in D} q(z)=\infty$, and let $p(z)=a+p_{1} z+\ldots$ be analytic in $D$ with $p(z) \neq a$. If there exists a point $z_{0} \in D$ such that $p\left(z_{0}\right) \in q(\partial D)$ and $p\left(|z|<\left|z_{o}\right|\right) \subset q(D)$. Then

$$
z_{0} p^{\prime}\left(z_{0}\right)=m \zeta q^{\prime}(\zeta),
$$

where $\mathrm{q}^{-1}\left(\mathrm{p}\left(\mathrm{z}_{\mathrm{o}}\right)\right)=\zeta=\mathrm{e}^{i \theta}$ and $\mathrm{m} \geqq 1$.

PROOF OF THEOREM 3. Let $g(z)=f(z) / z f^{\prime}(z), G(z)=k(\alpha, \beta, z) / z k^{\prime}(\alpha, \beta, z)$ and $H(z)=1 / G(z)$. The required result is equivalent to that

$$
g(z) \prec G(z)
$$

It is clear that (3.5) is to be the case if $g(z) \equiv 1$. So we assume that $g(z) \not \equiv 1$ next. We can easily check that

$$
\begin{aligned}
& 1+z f^{\prime \prime}(z) / f^{\prime}(z)=1 / g(z)-z g^{\prime}(z) / g(z), \\
& 1 / G(z)-z G^{\prime}(z) / G(z)=(1+(1-2 \beta \alpha) z) /(1+(1-2 \beta) z) .
\end{aligned}
$$

If (3.5) is not true, then by using Lemma 1 and Lemma $A$, there exists $z_{0}{ }^{\circ}$ such that

$$
z_{0} g^{\prime}\left(z_{0}\right)=m \zeta G^{\prime}(\zeta), g\left(z_{0}\right)=G(\zeta)
$$

where $|\zeta|=1$ and $m \geqq 1$. Thus we have

$$
\begin{aligned}
& 1+z_{o} f^{\prime \prime}\left(z_{o}\right) / f^{\prime}\left(z_{o}\right)=1 / G(\zeta)-m \zeta G^{\prime}(\zeta) / G(\zeta) \\
= & m\left(1 / G(\zeta)-\zeta G^{\prime}(\zeta) / G(\zeta)\right)-(m-1) / G(\zeta) \\
= & m(1+(1-2 \beta \alpha) \zeta) /(1+(1-2 \beta) \zeta)-(m-1) H(\zeta) .
\end{aligned}
$$

From Corollary 2, we know that $k(\alpha, \beta, z) \in S^{*}(\alpha, \beta)$, which gives that

$$
H(\zeta) \in \overline{Q(\alpha, \beta)} \text {. }
$$

For $\beta=1,(3.7)$ is equivalent to that $\operatorname{ReH}(\zeta) \geqq \alpha$. Thus (3.6) implies

$$
\begin{aligned}
& \quad \operatorname{Re}\left(1+z_{o} f^{\prime \prime}\left(z_{o}\right) / f^{\prime}\left(z_{o}\right)\right)=m \operatorname{Re}\{(1+(1-2 \alpha) \zeta) /(1-\zeta)\}-(m-1) \operatorname{ReH}(\zeta) \\
& \leqq \\
& m \alpha-(m-1) \alpha=\alpha,
\end{aligned}
$$

which contradicts that $f(z) \in K(\alpha, \beta)$.

For $\beta=\frac{1}{2},(3.7)$ becomes $|H(\zeta)-1| \leqq 1-\alpha$. And it follows from (3.6)

$$
\begin{aligned}
& \left|z_{\mathrm{o}} \mathrm{f}^{\prime \prime}\left(\mathrm{z}_{\mathrm{o}}\right) / \mathrm{f}^{\prime}\left(\mathrm{z}_{\mathrm{o}}\right)\right|=|\mathrm{m}(1+(1-\alpha) \zeta)-1-(\mathrm{m}-1) \mathrm{H}(\zeta)| \\
= & |\mathrm{m}(1-\alpha) \zeta-(\mathrm{m}-1)(\mathrm{H}(\zeta)-1)| \geqq \mathrm{m}(1-\alpha)-(\mathrm{m}-1)(1-\alpha)=1-\alpha,
\end{aligned}
$$


which is impossible since $f(z) \in K\left(\alpha, \frac{1}{2}\right)$.

For $B \neq \frac{1}{2}, 1,(3.7)$ is the same as

$$
|H(\zeta)-\alpha-(1-\alpha) / 2(1-\beta)| \leqq(1-\alpha) / 2(1-\beta) \text {. }
$$

We get from (3.6) that

$$
\begin{aligned}
& \left|1+z_{0} f^{\prime \prime}\left(z_{0}\right) / f^{\prime}\left(z_{0}\right)-\alpha-\frac{1}{2}(1-\alpha) /(1-\beta)\right| \\
= & \left|m(1+(1-2 \beta \alpha) \zeta) /(1+(1-2 \beta) \zeta)-\alpha-\frac{1}{2}(1-\alpha) /(1-\beta)-(m-1) H(\zeta)\right| \\
\geqq & m\left|(1+(1-2 \beta \alpha) \zeta) /(1+(1-2 \beta) \zeta)-\alpha-\frac{1}{2}(1-\alpha) /(1-\beta)\right| \\
- & (m-1)\left|\mathrm{H}(\zeta)-\alpha-\frac{1}{2}(1-\alpha) /(1-\beta)\right| \\
\geqq & m \frac{1}{2}(1-\alpha) /(1-\beta)-(m-1) \frac{1}{2}(1-\alpha) /(1-\beta)=\frac{1}{2}(1-\alpha) /(1-\beta),
\end{aligned}
$$

which is also impossible. This completes the proof of Theorem 3 .

For $\beta=1,(3.4)$ was first verified by MacGregor [7]. Our proof is much simpler than that in [7].

THEOREM 4. Let $f(z)=z+a_{2} z^{2}+\ldots$ be analytic in $D$. Then $f(z) \in K(\alpha, \beta)$ if and only if

$$
\frac{1}{z}\left\{f * \frac{z+z^{2}(\beta+\beta \alpha-1+x) / \beta(1-\alpha)}{(1-z)^{3}}\right\} \neq 0 \quad(|z|<1,|x|=1) .
$$

PROOF. We only prove the result for $\beta<1$. The result for $\beta=1$ can be deduced from that for $\beta<1$ by letting $\beta$ tend to 1 .

We know $f(z) \in K(\alpha, \beta)$ if and only if $1+z f^{\prime \prime}(z) / f^{\prime}(z) \in Q(\alpha, \beta)$ (z $\in$ D). Since $1+z f^{\prime \prime}(z) / f^{\prime}(z)=1$ at $z=0,1+z f^{\prime \prime}(z) / f^{\prime}(z) \in Q(\alpha, \beta)$ is equivalent to

$$
1+z f^{\prime \prime}(z) / f^{\prime}(z) \neq \alpha+\frac{1}{2}(1-\alpha)(1+y) /(1-\beta) \quad(|y|=1),
$$

which simplifies to

$$
z f^{\prime \prime}(z)+f^{\prime}(z) \frac{1}{2}(1-\alpha)(1-2 \beta-y) /(1-\beta) \neq 0 \quad(|y|=1) .
$$

As

$$
\begin{aligned}
& f^{\prime}(z)=\frac{f(z)}{z} * \frac{1}{(1-z)^{2}}, \\
& z f^{\prime \prime}(z)=\frac{f(z)}{z} * \frac{2 z}{(1-z)^{3}} .
\end{aligned}
$$

We have

$$
\begin{aligned}
& z f^{\prime \prime}(z)+f^{\prime}(z) \frac{1}{2}(1-\alpha)(1-2 \beta-y) /(1-\beta) \\
= & \frac{f(z)}{z} *\left\{2 z /(1-z)^{3}+(1-z)^{-2} \frac{1}{2}(1-\alpha)(1-2 \beta-y) /(1-\beta)\right\} \\
= & \frac{1}{2}(1-\alpha)(1-2 \beta-y)(1-\beta)^{-1} \frac{f(z)}{z} *\left\{\frac{1}{(1-z)^{3}}(1+(4(1-\beta) /(1-\alpha)(1-2 \beta-y)-1) z)\right\}
\end{aligned}
$$


It is not difficult to verify that $\frac{1}{2}(1-\alpha)(1-2 \beta-y) /(1-\beta) \neq 0$ and $2(1-\beta) /(1-2 \beta-y)=1-\frac{1}{2}(1-x) / \beta$ is a homotopic mapping from $|y|=1$ to $|x|=1$. Thus (3.8) is equivalent to

$$
\frac{f(z)}{z} * \frac{1+z(\beta+\beta \alpha-1+x) /(1-\alpha) \beta}{(1-z)^{3}} \neq 0 \quad(|x|=1),
$$

which is the same as the result desired. This completes the proof of Theorem 4.

For $\beta=1$, Theorem 4 was first given in [8].

4. APPLICATIONS.

With the help of principle of subordination, we can get the following results from Theorem 1 and Theorem 3. Here we omit their proof.

THEOREM 5. Let $\mathrm{f}(\mathrm{z}) \in \mathrm{K}(\alpha, \beta)$ and $|\mathrm{z}|=\mathrm{r}<1$, then we obtain sharp estimates.

$$
\begin{aligned}
& 2 \beta(1-\alpha) r /(1+|1-2 \beta| r) \leqq\left|z f^{\prime \prime}(z) / f^{\prime}(z)\right| \leqq 2 \beta(1-\alpha) r /(1-|1-2 \beta| r), \\
& \left|\arg \left(1+z f^{\prime \prime}(z) / f^{\prime}(z)\right)\right| \leqq \arcsin \left\{2 \beta(1-\alpha) r /\left(1-(1-2 \beta)(1-2 \beta \alpha) r^{2}\right)\right\} \text {, } \\
& (1+(2 \beta-1) r)^{2 \beta(1-\alpha) /(1-2 \beta)} \leqq\left|f^{\prime}(z)\right| \leqq(1-(2 \beta-1) r)^{2 \beta(1-\alpha) /(1-2 \beta)} \quad\left(\beta \neq \frac{1}{2}\right), \\
& e^{-(1-\alpha) r} \leqq\left|f^{\prime}(z)\right| \leqq e^{(1-\alpha) r} \quad \quad\left(\beta=\frac{1}{2}\right), \\
& |\operatorname{argf}(z)| \leqq(1-2 \beta)^{-1} 2 \beta(1-\alpha) \arcsin (1-2 \beta) r \quad\left(\beta \neq \frac{1}{2}\right), \\
& |\operatorname{argf}(z)| \leqq(1-\alpha) r \quad\left(\beta=\frac{1}{2}\right), \\
& \min _{|z|=r}\left|z k^{\prime}(\alpha, \beta, z) / k(\alpha, \beta, z)\right| \leqq\left|z f^{\prime}(z) / f(z)\right| \leqq r k^{\prime}(\alpha, \beta, r) / k(\alpha, \beta, r), \\
& \mid \arg \left\{z f^{\prime}(z) / f(z)\right\} \leqq \max _{|z|=r} \arg \left\{z k^{\prime}(\alpha, \beta, z) / k(\alpha, \beta, z)\right\} .
\end{aligned}
$$

Using a traditional method, we get from Theorem 5 the following

COROLLARY 3. Let $f(z) \in K(\alpha, \beta)$ and $|z|=r<1$, then we have sharp inequality

$$
-k(\alpha, \beta,-r) \leqq|f(z)| \leqq k(\alpha, \beta, r) \text {. }
$$

Theorem 3 also has an application of getting the sharp order of starlikeness for the functions in $K(\alpha, \beta)$.

COROLLARY 4. If $\mathrm{f}(\mathrm{z}) \in \mathrm{K}(\alpha, \beta),|\mathrm{z}|=\mathrm{r}<1$. Then

$$
\operatorname{Re}\left\{z f^{\prime}(z) / f(z)\right\} \geqq \min _{|z|=r} \operatorname{Re}\left\{z k^{\prime}(\alpha, \beta, z) / k(\alpha, \beta, z)\right\}
$$

In particular, we have $f(z) \in S^{*}(s(\alpha, \beta))$, where

$$
s(\alpha, \beta)=\inf _{|z|<1} \operatorname{Re}\left\{z^{\prime}(\alpha, \beta, z) / k(\alpha, \beta, z)\right\}>\alpha
$$

\section{REFERENCES}

1. OWA, S. A Note on Subordination, Internat. J. Math. \& Math. Sci. 9 (1986), 197-200.

2. JUNEJA, O.P. and MOGRA, M.L. On Starlike Functions of Order $\alpha$ and Type $\beta$, Rev. Roum. Math. Pures et App1. 13(1978), 751-765. 
3. MA, WANCANG On Starlike Functions of Order $\alpha$ and Type $B$, Kexue Tongbao 29(1984), 1404-1405; Pure and Applied Math. 2(1986), 35-43.

4. RUSCHEWEYH, S.T. and SHEIL-SMALL, T. Hadamard Products of Schlicht Functions and the Polya-Schoenberg Conjecture, Comment. Math. Helv. 48(1973), 119-135.

5. LIBERA, R.J. Some Classes of Regular Univalent Functions, Proc. Amer. Math. Soc. $16(1965), 755-758$.

6. MILLER, S.S. and MOCANU, P.T. Differential Subordinations and Univalent Functions, Michigan Math. J. 28(1981), 157-171.

7. MACGREgOR, T.H. A Subordination for Convex Functions of Order $\alpha$, J. London Math. Soc. 9 (1975), 530-536.

8. Silverman, H., Silvia, E.M. and TElaGE, D. Convolution Conditions for Convexity Starlikeness and Spira1-1ikeness, Math. Z. 162(1978), 125-130. 


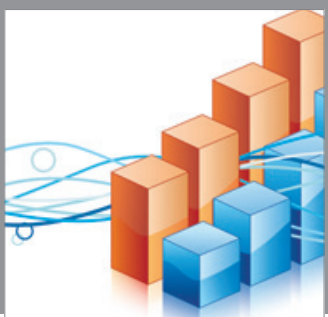

Advances in

Operations Research

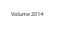

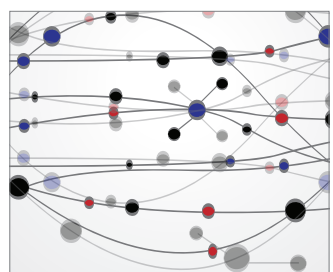

\section{The Scientific} World Journal
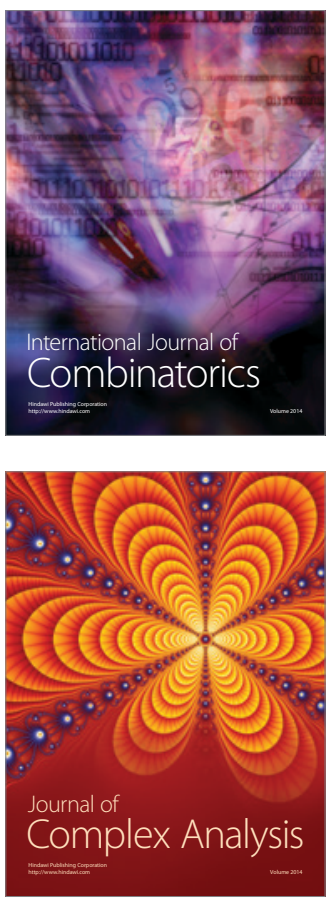

International Journal of

Mathematics and

Mathematical

Sciences
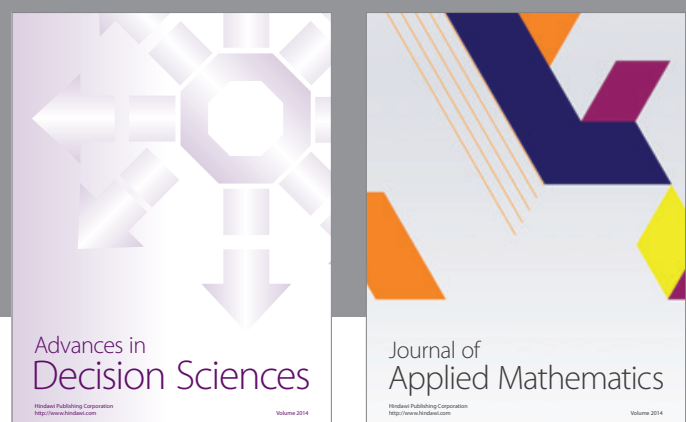

Journal of

Applied Mathematics
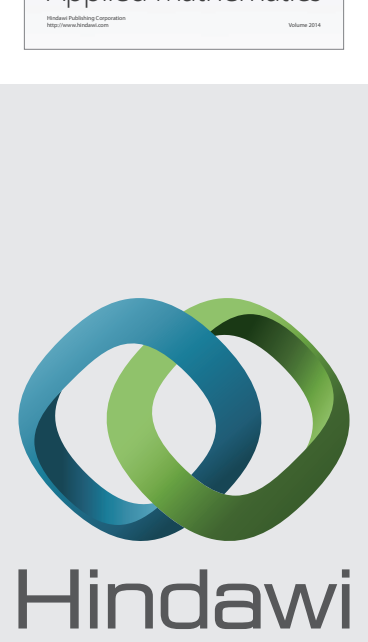

Submit your manuscripts at http://www.hindawi.com
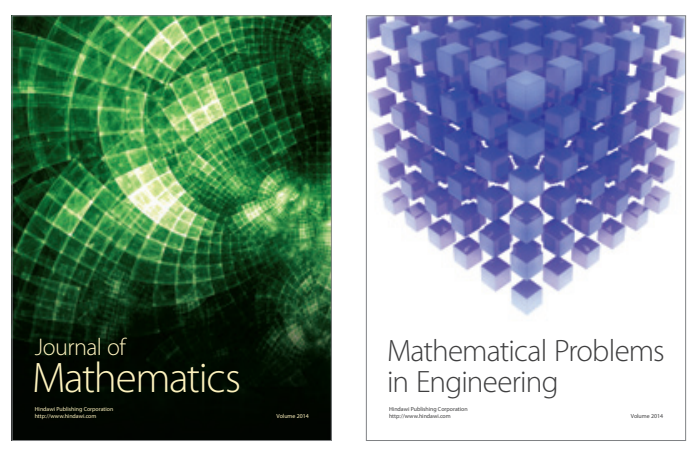

Mathematical Problems in Engineering
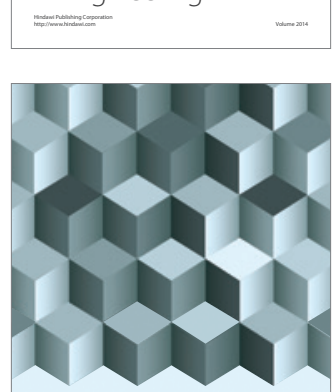

Journal of

Function Spaces
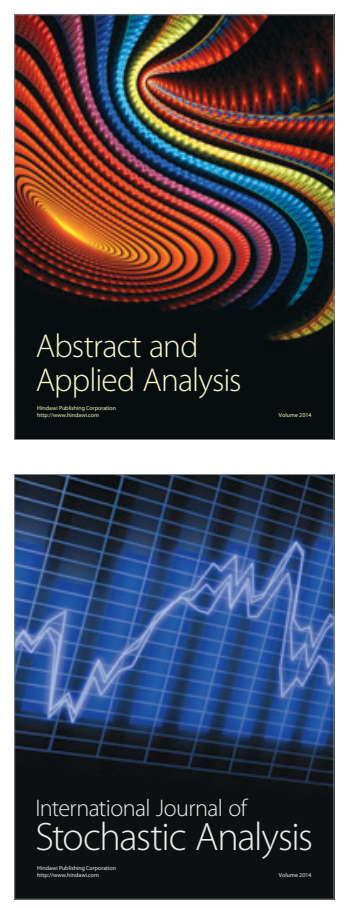

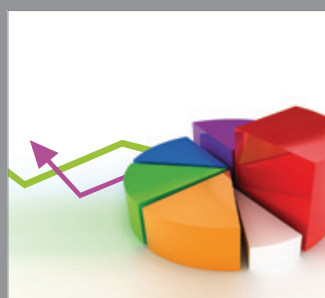

ournal of

Probability and Statistics

Promensencen
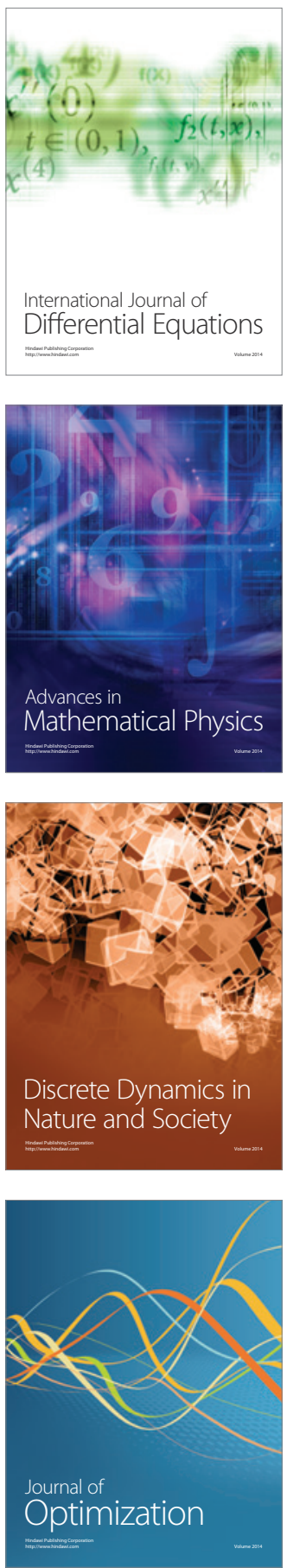